\title{
PROPIEDAD INTELECTUAL, LIBRE COMPETENCIA Y BIENES PÚBLICOS: $U N$ ANÁLISIS DESDE LAS REGLAS DE LA CATEDRAL
}

Mario Andrés Pinzón Camargo

Máster en Derecho Económico, Universidad Externado de Colombia. Maestría en Políticas Públicas de la Universidad Nacional de Colombia. Profesional en Gobierno y Relaciones Internacionales de la Universidad Externado de Colombia. Docente - Investigador en el Departamento de Derecho Económico,

Universidad Externado de Colombia.

Correo electrónico: mario.pinzon@uexternado.edu.co

\begin{abstract}
Resumen
El sistema de propiedad intelectual es considerado como un arreglo institucional por medio del cual se busca equilibrar la necesidad de recuperar la inversión en trabajo de un agente innovador y por otro aumentar el bienestar de la sociedad en cuanto al acceso a nuevo conocimiento, sin embargo, la estructura contemporánea de este sistema en lugar de lograr el equilibrio mencionado, ha provocado fuertes distorsiones en la libre competencia las cuales afectan negativamente el bienestar del consumidor al perder en gran medida parte de su justificación natural y dar pie a repensar su configuración interna. Con el objetivo de analizar la anterior afirmación este documento se encuentra compuesto por cuatro apartados. El primero analiza el conocimiento como un bien público, da cuenta de sus características básicas y problemas asociados. El segundo desarrolla una aproximación al sistema de propiedad intelectual, evidencia las características básicas de est a institución; El tercero, efectúa algunas críticas a su diseño institucional. Finalmente se esgrimen algunos pilares para una nueva arquitectura intelectual.
\end{abstract}

Palabras clave: Propiedad Intelectual; Innovación y Desarrollo; Competencia; Protección del consumidor

JEL O31; O34; D18

\begin{abstract}
The intellectual property system is considered as an institutional arrangement by which it seeks to balance the need to recover the investment in a work by another innovator and improve the welfare of society in terms of access to new knowledge. However, the contemporary structure of this system instead of achieving the mentioned balance,, has led to major distortions in competition which adversely affect consumer welfare, certainly losing much of its natural justification, and giving rise to rethink their internal configuration. In order to analyze the statement above this document is organized in four sections. The first analyzes the knowledge as a public good, highlighting their basic characteristics and associated problems. The second section develops an approach to intellectual property system, demonstrating the basic features of this institution; subsequently some criticisms of its institutional design are exposed. Finally some pillars for a new intellectual architecture is put forward.
\end{abstract}

Keywords: Intellectual property; Development \& Innovation; Competition; Consumer protection

\section{Résumé}

Le système de propriétéintellectuelleestconsidérécomme un accord institutionnel au moyenduquelcherche à êtreéquilibrée la nécessité de récupérerl'investissementdans travail d'un agent innovateur et la structure contemporaine de cesystèmeaugmente pour l'autre le bien-être de la société en ce qui concernel'accès à une nouvelle connaissance, cependant, au lieu d'obtenirl'équilibrementionné, a provoqué de fortes distorsionsdans la libre concurrence lesquellesaffectentnégativement le bien-être du consommateur après avoirperdu en grand partieune dépêche de sa justification naturelle et après avoirdonné un pied à repensersa configuration interne. Avec l'objectifd'analyserl'affirmationantérieurece document trouvecomposé par quatre parties. Le premier analyse la connaissancecommeunbien public, se rend compte de sescaractéristiquesbasiques et de problèmesassociés. Le deuxièmedéveloppeuneapproche au système de propriétéintellectuelle, met en évidence les caractéristiquesbasiques de cette institution; Le troisième, effectuequelques critiques à son dessininstitutionnel. On affirmefinalementquelquesbornes pour une nouvelle architecture intellectuelle.

Mots-clés: la PropriétéIntellectuelle; l'Innovationet le Développement; la Concurrence; la Protection du consommateur 

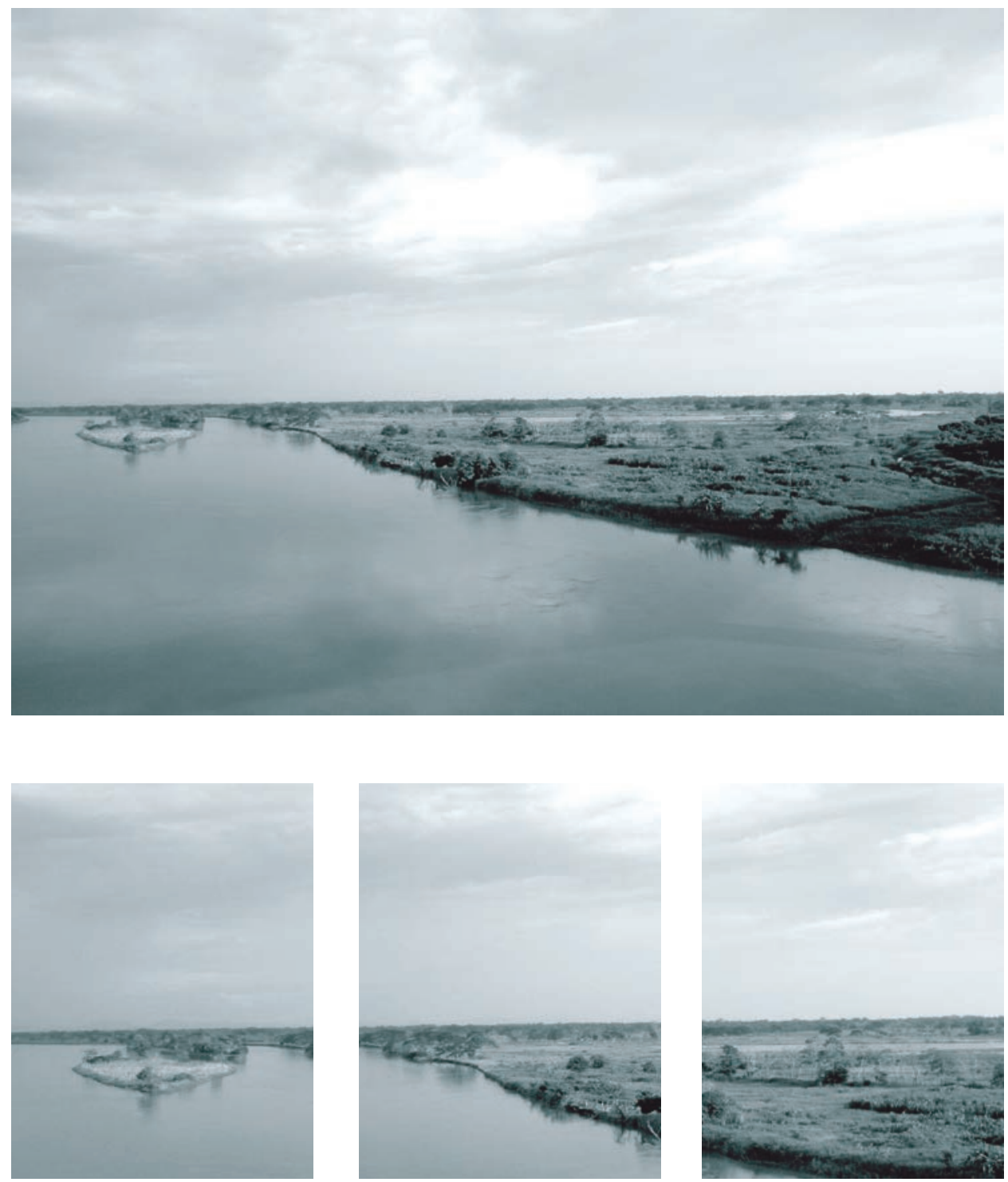

Rio Magdalena - Mompóx - Colombia

Martín Emilio Hernández Manrique 


\section{PROPIEDAD INTELECTUAL, LIBRE COMPETENCIA Y BIENES PÚBLICOS: $U N$ ANÁLISIS DESDE LAS REGLAS DE LA CATEDRAL *}

Mario Andrés Pinzón Camargo

Las mezquitas caen, los palacios se convierten en polvo, pero el conocimiento permanece. UlugBeg

\section{El conocimiento como un bien público: Características y problemas}

Para nadie es un secreto que los cambios sociales, políticos y económicos que la sociedad ha enfrentado en el último cuarto del siglo XX en gran medida se encuentran explicados por una elevada tasa de cambio tecnológico. De hecho hoy en día se afirma que la fuente de riqueza de un país no se encuentra en sus factores productivos tradicionales (tierra, capital y trabajo) sino en su capacidad científica y tecnológica. Lo anterior en cuanto que, como lo presentan Samuelson y Nordhaus (1999, p. 514), a diferencia de los factores productivos que tienen rendimientos decrecientes a escala, al implementar avances en ciencia y tecnología la frontera de productividad se desplaza, reflejando con ello el aumento de la capacidad productiva de los factores tradicionales. Esto se puede ver en el Gráfico 1.

* Texto producto de la Investigación: Análisis Crítico al sistema de propiedad intelectual: una aproximación desde el AED y las políticas públicas. Departamento de Derecho Económico, Universidad Externado de Colombia. Agradezco las valiosas observaciones y comentarios del profesor Daniel Monroy, docente investigador del Departamento de Derecho Económico de la Universidad Externado de Colombia. 
Gráfico 1. Crecimiento de la productividad por efecto del cambio tecnológico

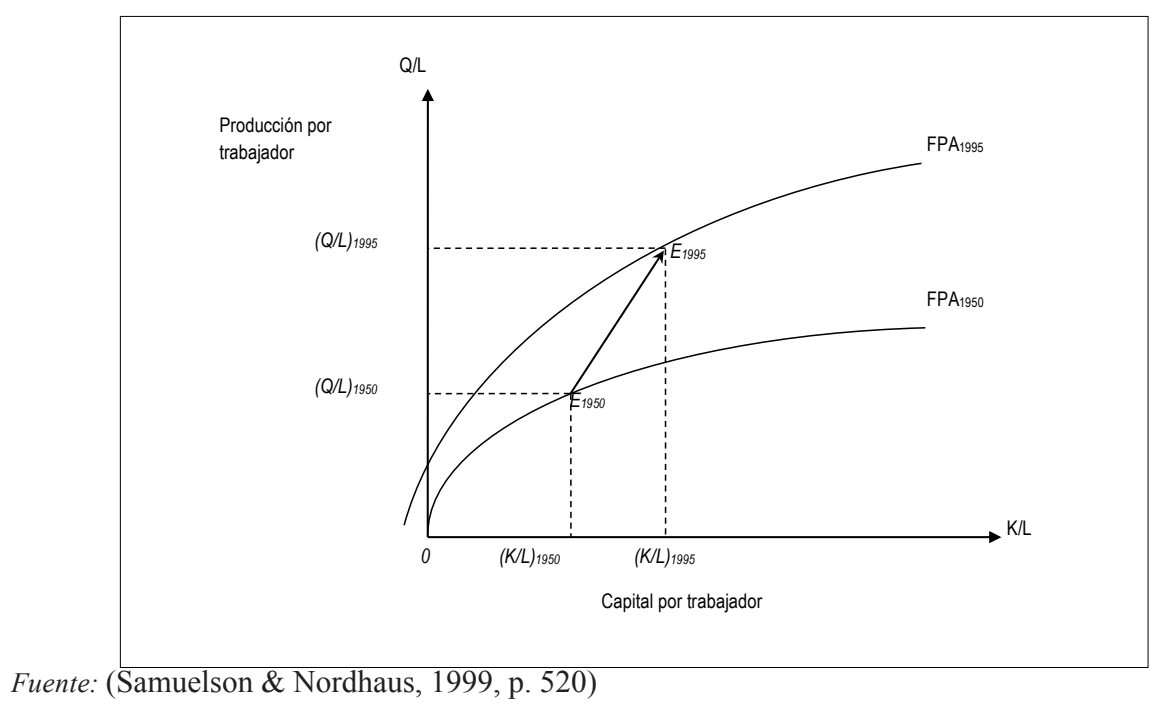

En el Gráfico 1, la diferencia entre los puntos $\mathrm{E}_{1950}$ y $\mathrm{E}_{1995}$ que es representada por una flecha, ilustra el aumento en el rendimiento de los factores productivos. Rendimiento que, a su vez, implica un aumento de los niveles de productividad de un país o una región y que, a la postre, implicará un aumento del bienestar social. Esto es ratificado por autores como Mankiw, para quien "[1]a razón principal por la que los niveles de vida son más altos hoy se halla en que los conocimientos tecnológicos han avanzado" (1998, p. 344).

Lo anterior conduce a pensar que (...) [d]e la misma manera que el Estado desempeña un papel importante en la provisión de un bien público como la defensa nacional, también desempeña un papel importante en el fomento de la investigación y el desarrollo de nuevas tecnologías" (Mankiw, 1998, p. 344), bajo el entendido que es la base para el cambio tecnológico y la generación de condiciones para aumentar el bienestar social.

Es en este escenario donde la tecnología se presenta como una fuente estratégica para el desarrollo de las naciones. En razón a lo cual es innegable la necesidad de intervención del Estado a través de la definición de derechos de propiedad, sistemas contractuales y, en general, del marco institucional necesario para garantizar que los efectos directos como las externalidades positivas que se derivan de los avances en ciencia y tecnología sean trasladados a los consumidores y, en general, a toda la sociedad y que, a su vez, las externalidades y los efectos directos negativos sean neutralizados o al menos minimizados. 
Comprender un poco mejor el papel que desempeña el Estado en todo es proceso de promoción de la ciencia y la tecnología, la innovación, la investigación y el desarrollo lo que implica, necesariamente, detenerse en el estudio de dos aspectos, el primero de ellos corresponde al estudio del bien sobre el cual subyacen todas las acciones del Estado en las áreas y los sectores mencionados, es decir, en el conocimiento; y segundo, analizar la estructura institucional ideada para proteger e incentivar la producción de este bien tan importante para la sociedad.

Desde el punto de vista de la teoría económica de los derechos de propiedad, el conocimiento se puede catalogar como un bien público ${ }^{1}$, en cuanto que no es rival, es decir, que el hecho que alguien use un conocimiento determinado no afecta el uso potencial que un tercer agente puede hacer sobre el mismo tipo de conocimiento; a su vez, no es excluyente en cuanto que no es posible relegar de su uso o consumo a terceros (Mankiw, 1998, p. 140). Ahora bien, resulta pertinente aclarar que esta clasificación sólo puede efectuarse bajo el supuesto que no existen reglas jurídicas que modifiquen artificialmente las condiciones de los derechos de propiedad que definen el conocimiento o, en otras palabras, que no existen reglas de propiedad intelectual o que éstas no aplican.

En este orden y bajo el entendido que el conocimiento es un bien público, surgen una serie de inconvenientes en relación a este tipo de bienes. Como lo explica Stiglitz (2000) los bienes públicos son catalogados dentro de un conjunto de fenómenos denominados como fallas del mercado. En este caso la falla se relaciona con la provisión óptima de este bien en cuanto que al no ser posible excluir de su consumo o uso a terceros, no se generan incentivos para que el empresario innovador o para el agente innovador provea dicho bien, puesto que no podrá ver compensada su inversión en trabajo y/o en capital.

Sumado a lo anterior, bajo el entendido que el agente innovador tome la determinación de proveer el bien público al mercado, la imposibilidad de exclusión configurará las condiciones óptimas para la aparición de un comportamiento estratégico negativo como lo es el del free rider o polizón. Lo anterior implicaría que los costos de transacción asociados a la vigilancia para el agente innovador en relación al uso por parte de terceros serían muy altos, siendo posible la aparición de acciones como la elusión o la evasión de pagos.

Ahora bien, como resulta evidente, una producción sub-óptima de conocimiento implicará un nivel inadecuado de ciencia y tecnología, lo cual llevaría a una disminución en la tasa de cambio tecnológico y con ello de las externalidades positivas en materia de bienestar social. En este marco, como se mencionó, se justifica la acción del Estado orientada a corregir la falla del mercado asociada a los bienes públicos.

1 A su vez, los bienes públicos pueden ser definidos como “(...) aquellos que benefician de una manera indivisible a toda la comunidad, independientemente que los individuos deseen o no comprarlos"(Samuelson \& Nordhaus, 1999, p. 336) 
La corrección de esta falla se materializa por medio de la creación de una estructura institucional conocida como el sistema de propiedad intelectual, que como se ya se anunció, ha sido ideado con la finalidad de proteger e incentivar la producción de conocimiento. Para ello, a través de este sistema el Estado modifica las reglas de propiedad aplicables al conocimiento, con lo que pasa de ser un bien público a asemejarse a un bien privado, caracterizándose entonces ahora, por efecto de este cambio, por ser rival y excluyente.

En relación a la propiedad intelectual, es pertinente mencionar, como lo señalan Buitrago \& Castañeda Lozano (2011) en consideración a las Sentencias C-334 de 1993 y C-871 de 2010 de la Corte Constitucional, que ésta es considerada como

“(...) un tipo de propiedad sui generis, en la medida en que en la sentencia C-871 de 2010, al reiterar la línea jurisprudencial contenida en la sentencia C-334 de 1993, la H. Corte Constitucional trascribe los apartes de dicho fallo en que se indica que se trata de un tipo de propiedad con características especiales" (2011, p. 4).

Al comparar las diferencias entre la propiedad común y la propiedad intelectual, los autores mencionados señalan que en consideración a cinco criterios examinados por la Corte Constitucional en las sentencias analizadas, es posible encontrar diferencias y semejanzas señaladas en el Cuadro 1.

Cuadro 1. Propiedad intelectual vs. Propiedad común: diferencias y semejanzas

\begin{tabular}{lcc}
\hline \multicolumn{1}{c}{ Elemento de comparación } & Propiedad intelectual & Propiedad común \\
\hline $\begin{array}{l}\text { Existen los elementos esenciales de la propiedad } \\
\text { iusutendi, fruendi y abutendi }\end{array}$ & Sí & Sí \\
\hline El derecho tiene un contenido patrimonial & Sí & Sí \\
\hline El derecho patrimonial dura a perpetuidad & No, es temporal & Sí \\
\hline $\begin{array}{l}\text { El autor tiene contenido moral del derecho que es } \\
\text { inalienable, irrenunciable e imprescriptible. Además } \\
\text { es independiente del contenido patrimonial }\end{array}$ & Si & $\begin{array}{c}\text { No tiene } \\
\text { contenido moral. }\end{array}$ \\
\hline Recae sobre cosas incorporales & Sí & $\begin{array}{l}\text { NO, recae sobre } \\
\text { cosas corporales }\end{array}$ \\
\hline
\end{tabular}

Fuente: (Buitrago \& Castañeda Lozano, 2011, p. 3)

Del Cuadro 1 se puede concluir que la propiedad intelectual se caracteriza, o se diferencia de la propiedad común por tener una temporalidad definida, la cual usualmente responde al cumplimiento de una función social que este derecho debe cumplir, concretándose en la extinción del derecho de explotación exclusivo, para 
que el conocimiento que se protege pase al dominio público2; así como también se caracteriza por recaer sobre bienes incorporales y poseer un contenido moral. Por su parte, en relación a las similitudes con la propiedad común se encuentra que comparten la existencia de los elementos esenciales de la propiedad y la existencia de un derecho patrimonial.

Hasta este punto se evidencia cómo el sistema de propiedad intelectual se constituye como un arreglo institucional por medio del cual el Estado pretende resolver una falla de mercado como lo son los bienes públicos, bajo el entendido “(...) que al protegerse la invención se estimula a aquél o aquéllos que quieren realizar inventos, y que de esa manera se contribuye al desarrollo y a la evolución tecnológica de la sociedad" (Castro García, 2009, pp. 243-244) y, a su vez, se evita la aparición de agentes oportunistas que deseen beneficiarse del trabajo innovador sin haber aportado de ninguna manera. Se reitera entonces que el objetivo de este arreglo institucional no es otro que cambiar las características de un bien público para convertirlo en un bien sobre el cual se pueda ejercer la capacidad de exclusión.

\section{Una aproximación al sistema de propiedad intelectual: características de esta institución}

El segundo punto abordado para tratar de comprender el papel que desempeña el Estado en el proceso de promoción de la ciencia y la tecnología, la innovación, la investigación y el desarrollo se centra en el estudio de la estructura institucional ideada para proteger e incentivar la producción de este bien tan importante para la sociedad, la propiedad intelectual. Para ello se tomará como caso de estudio la Decisión 486 de 2000 del Comunidad Andina (en adelante la Decisión) y el Anexo 1C del Acuerdo de la Organización Mundial del Comercio (OMC) más conocido como el acuerdo sobre los Aspectos Derechos de Propiedad Intelectual relacionados con el Comercio (ADPIC), y de estos el sistema de patentes, el cual pertenece a un subsistema denominado como propiedad industrial y que, conjuntamente con los derechos de autor, conforman los dos grandes grupos que conforman el arreglo institucional.

En este orden, las patentes pueden ser definidas “(...) como el derecho de propiedad asignado sobre un bien inmaterial a través del cual se justifica la explotación del mismo por parte del inventor, por medio de una estructura monopolística, en un horizonte de tiempo definido" (Pinzón Camargo, 2010, p. 189), o en otras palabras, el

2 Se debe resaltar que a pesar que usualmente la función social de la propiedad intelectual se asocia con la liberación del conocimiento al dominio público, luego de periodos determinados bajo criterios políticos y no técnicos, es necesario revaluar si con esta acción de disponer la información en el dominio público se cumple realmente con la función que mencionaba. Máxime cuando ésta presupone un criterio para equilibrar el beneficio privado con el beneficio público. 
“(...) privilegio que le otorga el Estado al inventor como reconocimiento de la inversión y esfuerzos realizados por éste para llegar a la invención que aporta una solución técnica a la humanidad. Dicho privilegio consiste en el derecho a explotar exclusivamente el invento por un tiempo determinado" (SIC, 2008).

A su vez, las patentes se encuentran divididas en dos categorías. En primera instancia se encuentran las patentes de modelo de utilidad, las cuales tiene una protección de 10 años y se asignan a

“(...) aquellas invenciones que consisten en una nueva forma, configuración o disposición de elementos de un artefacto, herramienta, instrumento, mecanismo $\mathrm{u}$ otro objeto o parte de los mismos, que permita un mejor o diferente funcionamiento, utilización o fabricación del objeto que lo incorpora o que le proporcione alguna utilidad, ventaja o efecto técnico que antes no tenía" (SIC, 2008).

Como segunda categoría se encuentran las patentes de innovación, las cuales están conformados

“(...) por todas las invenciones, sean de productos o de procedimientos, en todos los campos de la tecnología, siempre que sean nuevas, entrañen una actividad inventiva y sean susceptibles de aplicación industrial" (OMC, 1994), para este caso la protección será de 20 años.

Tomadas como marco de análisis las reglas propuestas por Calabresi \& Melamed en su artículo Reglas de propiedad, reglas de responsabilidad y de inalienabilidad: una vista de la catedral (1972), resulta posible estructurar el análisis del sistema de patentes en relación al problema de la asignación inicial de los derechos de propiedad, y posteriormente a la verificación de la aplicación de las reglas propuestas por estos dos autores.

Así, en relación a la asignación inicial del derecho de propiedad intelectual, que en este caso se concreta en la patente sobre el conocimiento innovador, se debe partir por plantear que un derecho de propiedad se define como

"(...) un conjunto de derechos, los cuales describen lo que los individuos pueden o no hacer con los recursos de su propiedad: la medida que pueden poseer, usar, transformar, transferir o excluir a otros de su propiedad" (Cooter \& Ulen, 1997, p. 104);

o de manera general como lo plantean Furobont y Pejovich, los derechos de propiedad son

“(...) el conjunto de relaciones económicas y sociales que definen la posición de cada individuo con respecto del uso de recursos escasos" (1972, p. 1138)

3 Texto original en inglés: “(...) the set of economic and social relations defining the position of each individual with respect to the utilization of scarce resources" (traducción del autor). 
La manifestación más clara de las definiciones presentadas es posible encontrarlas en los artículos 27 a 34 del acuerdo ADPIC. Se observa por ejemplo cómo el artículo 28 de este acuerdo determina que:

1. Una patente conferirá a su titular los siguientes derechos exclusivos:

a) cuando la materia de la patente sea un producto, el de impedir que terceros,

b) sin su consentimiento, realicen actos de: fabricación, uso, oferta para la venta, venta o importación para estos fines del producto objeto de la patente;

c) cuando la materia de la patente sea un procedimiento, impedir que terceros, sin su consentimiento, realicen el acto de utilización del procedimiento y los actos de: uso, oferta para la venta, venta o importación para estos fines de, por lo menos, el producto obtenido directamente por medio de dicho procedimiento.

2. Los titulares de patentes tendrán asimismo el derecho de cederlas o transferirlas por sucesión y de concertar contratos de licencia.

Se destaca cómo en el anterior artículo se muestra claramente cómo se faculta a los agentes titulares del conocimiento para ejercer la capacidad de exclusión, no por las características propias del bien, sino por efecto de la regla jurídica, con lo cual sería posible pensar que se generan incentivos para producir el nivel óptimo de conocimiento útil, así como para neutralizar la acción de los free riders. De manera similar opera la Decisión en sus artículos 50 a 58.

Sin embargo, para que estas reglas puedan aplicarse, el conocimiento a ser protegido debe cumplir con tres requisitos definidos tanto en el artículo 14 de la Decisión, como en el numeral 1 del artículo 27 del acuerdo ADPIC. Así, “(...) las patentes podrán obtenerse por todas las invenciones, sean de producto o de procedimiento, en todos los campos de la tecnología, siempre que sean nuevas, tengan nivel inventivo y sean susceptibles de aplicación industrial" (OMC, 1994, Art. 27).

Sumado a lo anterior, el artículo 22 de la Decisión, determina que:

Artículo 22.- El derecho a la patente pertenece al inventor. Este derecho podrá ser transferido por acto entre vivos o por vía sucesoria. Los titulares de las patentes podrán ser personas naturales o jurídicas. Si varias personas hicieran conjuntamente una invención, el derecho a la patente corresponde en común a todas ellas.

Si varias personas hicieran la misma invención, independientemente unas de otras, la patente se concederá a aquella o a su causahabiente que primero presente la solicitud correspondiente o que invoque la prioridad de fecha más antigua. (Negrilla fuera de texto) 
Se observa que la asignación inicial del derecho es efectuada al inventor, sin embargo, se advierte cómo, a manera de incentivo a la competencia, la asignación se condiciona a la primera solicitud que reivindique la titularidad del derecho ${ }^{4}$. Con este mecanismo se soluciona el problema de la titularidad inicial del derecho. Así, se observa que la titularidad del derecho de propiedad de intelectual se encuentra en función de una variable de orden temporal asociada a la solicitud de la patente; y una variable de tipo cualitativo ligada a las características que debe cumplir el conocimiento para que sea objeto de protección.

Definida la regla para la asignación inicial del derecho de propiedad intelectual corresponde ahora constatar el uso de las tres reglas que proponen Calabresi y Melamed (1972). Para ello se parte por definir cada una de las reglas que se proponen, para de allí dar paso al análisis del diseño de este derecho de propiedad, el cual como ser verá responder a la lógica arquitectónica propuesta por estos dos autores.

En este orden se puede decir, que

"[u]n derecho es protegido por una regla de propiedad en cuanto que quien desea apoderarse de un derecho en cabeza de otro titular debe pagar por este a través de una transacción voluntaria en la cual el valor del derecho es aceptado por el vendedor. Esta es la forma de titulación que permite el menor nivel de intervención del Estado: una vez la asignación inicial es decidida, el Estado no entra a definir su valor" (Calabresi \& Melamed, 1972, p. 1092)

Una regla de responsabilidad aplica si alguien que puede destruir un derecho inicial está dispuesto a pagar por éste un valor determinado objetivamente. Este valor puede ser que el que pensó el tenedor original del derecho que podría haber obtenido por su venta. (...) Obviamente, las reglas de responsabilidad implican un nivel superior de intervención estatal: no solamente protegen los derechos, sino también permite su transferencia o destrucción con base en un valor determinado por algún

4 Un caso que evidencia este tipo de incentivos a la competencia se puede observar del proceso de investigación del genoma humano. Allí existían dos grupos de investigación, uno financiado a través de fondos públicos y otro por recursos privados. En este ejemplo, la reivindicación de la titularidad de la patente implicaba la posibilidad de generar barreras de acceso al conocimiento derivado del proceso de investigación en el caso que el grupo más eficiente fuese aquel patrocinado por fondos privados, frente a la divulgación de información en el dominio público si el grupo de investigación financiado con fondos públicos lo lograra. El incentivo en este caso llevó a que el grupo de investigación con respaldo en fondos públicos terminara primero el proyecto del genoma humano, beneficiando con ello a la humanidad, al disponer de un banco de datos e información de gran utilidad para la investigación en diferentes campos de las ciencias de la Salud.

5 Texto original en inglés: "An entitlement is protected by a property rule to the extent that someone who wishes to remove the entitlement from its holder must buy it from him in a voluntary transaction in which the value of the entitlement is agreed upon by the seller. It is the form of entitlement which gives rise to the least amount of state intervention: once the original entitlement is decided upon, the state does not try to decide its value" (Traducción libre del autor). 
órgano estatal en lugar de sus partes (Calabresi \& Melamed, 1972, p. 1092) ${ }^{6}$, como en el caso de la regla de propiedad antes presentado.

Finalmente, se encuentran la regla de inalienabilidad, la cual implica el nivel más alto intervención estatal, y "se puede soportar en razones de eficiencia y/o distribución" (Monroy Cely, 2010, p. 30). Para que esta regla pueda ser aplicada es necesario que no sea posible la transacción del derecho, incluso bajo la idea de una compensación por una vulneración de éste. Lo anterior en atención a que de permitirse la transacción las externalidades negativas que ésta produce son significativas y, a su vez, se presentan al menos una de las dos siguientes situaciones:

"Primero, el valor superior de los costos sociales en sí asociados a la magnitud de los afectados con una conducta determinada (el tamaño de la sociedad como tal) y, segundo, la dificultad de establecer ex-ante criterios objetivos que permitan hacer tal medición" (2010, p. 29).

Al contrastar las tres reglas mencionadas con el sistema de patentes se puede encontrar a la luz de la regla de propiedad la existencia de dos tipos de transacciones derivadas de la forma como se ha configurado el sistema de patentes. El primer tipo de transacciones corresponde a aquellas que surgen en ocasión de la facultad que tiene el agente innovador para conceder vía licencia la explotación de su trabajo, tal cual como se encuentra definido en el artículo 57 de la Decisión.

"[e]l titular de una patente concedida o en trámite de concesión podrá dar licencia a uno o más terceros para la explotación de la invención respectiva".

En este caso la licencia implica una negociación entre quien desea tener la licencia de explotación y quien tiene la facultad de concederla, mediada por un precio materializado en el pago de una regalía al titular de la patente.

El segundo tipo de transacciones corresponde a un intercambio donde además de la posibilidad de explotación de la patente se efectúa la transferencia de la capacidad de uso y abuso del derecho de propiedad intelectual. Se habla entonces de una transferencia casi plena de la propiedad intelectual, que en esta oportunidad se concreta en la compra de la patente. No se puede afirmar que sea plena en cuanto que como se analizará adelante, persisten los derechos morales asociados al inventor.

La segunda regla de que se ocupan Calabresi y Melamed corresponde a la regla de responsabilidad. De manera similar a la regla anterior, en este caso la aplicación de esta regla se puede ver de manera clara frente a dos tipos de situaciones concretas.

$6 \quad$ Texto original en inglés: "Whenever someone may destroy the initial entitlement if he is willing to pay an objectively determined value for it, an entitlement is protected by a liability rule. This value may be what it is thought the original holder of the entitlement would have sold it for. But the holder's complaint that he would have demanded more will not avail him once the objectively determined value is set. Obviously, liability rules involve an additional stage of state intervention: not only are entitlements protected, but their transfer or destruction is allowed on the basis of a value determined by some organ of the state rather than by the parties themselves" (Traduccion libre del autor). 
La primera de ellas corresponde a prácticas como la piratería y la falsificación ${ }^{7}$. Allí claramente se encuentra una situación donde un producto es copiado o vendido sin el permiso del propietario legítimo (Balkin, Shepherd, \& De Castro, 2004) de los derechos de propiedad intelectual. Esta regla se encuentra expresada en el artículo 239 de la Decisión.

El titular de una patente tendrá derecho a ejercer acción judicial por daños y perjuicios por el uso no autorizado de la invención o del modelo de utilidad durante el período comprendido entre la fecha en que adquiera carácter público y pueda ser consultada la solicitud respectiva y la fecha de concesión de la patente. El resarcimiento sólo procederá con respecto a la materia cubierta por la patente concedida, y se calculará en función de la explotación efectivamente realizada por el demandado durante el período mencionado.

En este caso la destrucción o vulneración del derecho de propiedad del que hablan Calabresi y Melamed se cristaliza en la acción de copiar, vender, usar y/o explotar sin autorización el bien que es protegido por el derecho de propiedad intelectual. Frente a esta situación el titular del derecho de propiedad responde a través de una demanda por medio de la cual busca detener los actos que están vulnerando su derecho y de otra parte que le sea resarcido el daño económico que este tipo de comportamiento le ha generado y que es cuantificado a través de estudios de mercado. Como se presenta en la definición de esta regla, en este caso el papel del Estado además de definir la asignación inicial de los derechos de propiedad intelectual, se presenta a través decisión judicial por medio de la cual busca simular el resultado que el mercado hubiese alcanzado en el caso de haberse aplicado una regla de propiedad.

El segundo escenario donde se evidencia el uso de la regla de responsabilidad se manifiesta en el uso de licencias obligatorias, las cuales se pueden asimilar con un procedimiento de expropiación. En este caso el derecho es vulnerado por parte del Estado en atención a circunstancias claramente definidas como se puede evidenciar en la Decisión, en relación a tres circunstancias: cuando luego de tres años de haber concedido la patente o de cuatro de haber hecho la solicitud de la misma, el titular del derecho no se hubiere explotado la patente en los términos de los artículos 59 y 60 de la misma Decisión (Art. 61); cuando previa declaratoria de un País Miembro de razones de interés público, de emergencia, o de seguridad nacional, y mientras estas razones permanezcan (Art. 65); finalmente, cuando se presenten prácticas que afecten la libre competencia, en particular, cuando constituyan un abuso de la posición dominante en el mercado por parte del titular de la patente (Art. 66).

7 Acorde con Balkin, Shepherd, \& De Castro (2004) existe una diferencia entre piraterÍa y falsificación. En este orden mientras la piratería corresponde a aquellas transacciones donde existe plena de conciencia por parte de quien desea comprar el producto respecto a que el tipo de producto es ilegal o pirata, bien sea por el lugar donde lo adquiere o por el precio del producto; en el caso de la falsificación existe un engaño deliberado hacia el consumidor, quien incurre en una compra de un producto que considera legal pero que en realidad corresponde un producto pirata. 
Conjuntamente con la descripción de las situaciones bajo las cuales se contempla la destrucción o vulneración del derecho de propiedad intelectual, se encuentra el reconocimiento explícito de la compensación de orden económico en que se debe incurrir por tal acción. Muestra de ello es el artículo 62 de la Decisión, en cuyo inciso 2 se contempla que:

La oficina nacional competente establecerá el alcance o extensión de la licencia, especificando en particular, el período por el cual se concede, el objeto de la licencia, el monto y las condiciones de la compensación económica. Esta compensación deberá ser adecuada, según las circunstancias propias de cada caso, considerando en especial el valor económico de la autorización.

Acorde con la idea de compensación económica del artículo 62 mencionado, pero en este caso reconociendo que la licencia se genera con ocasión de una práctica anticompetitiva el artículo 66 de la Decisión determina los procedimientos para calcular la compensación económica en el parágrafo segundo:

En estos casos, para determinar el importe de la compensación económica, se tendrá en cuenta la necesidad de corregir las prácticas anticompetitivas.

La idea de la compensación económica en ocasión a la vulneración del derecho de propiedad que implica la licencia obligatoria, es reconocida nuevamente en el literal f del artículo 67 de la Decisión, donde se ordena que la licencia estará sujeta, sin perjuicio de lo dispuesto en el artículo 68 de la misma, a lo siguiente:

(...) contemplará una remuneración adecuada según las circunstancias de cada caso, habida cuenta del valor económico, sin perjuicio de lo dispuesto en el artículo 66;

Así, al igual que en el caso de la piratería, las licencias obligatorias configuran la aplicación de una regla de responsabilidad en los términos expuestos por Calabresi y Melamed, cuando a pesar de existir un derecho de propiedad, este no es transado de manera voluntaria sino que es sometido a una vulneración que necesariamente implica un pago orientado a resarcir el daño generado. El cual puede ser calculado de manera objetiva a través de, por ejemplo, la estimación de las regalías perdidas en caso que hubiese operado una regla de propiedad asociada a la emisión de licencias; la estimación de los recursos que no se pudieron percibir en el caso de haber vendido la patente, es decir, la aplicación de la segunda regla de propiedad mencionada anteriormente; o incluso el cálculo de los ingresos no percibidos por la explotación directa del conocimiento objeto de protección de la patente.

Como última regla en relación a la propiedad intelectual se encuentra la regla de inalienabilidad. En este caso se podría presumir que la idea de la paternidad de la innovación la cual se ve plasmada en el artículo 24 de la Decisión: El inventor tendrá derecho a ser mencionado como tal en la patente y podrá igualmente oponerse a esta mención. 
Esta paternidad se asocia con la existencia de un derecho moral para el agente innovador, el cual se caracteriza porque "(...) es inalienable, irrenunciable e imprescriptible. Además es independiente del contenido patrimonial” (Buitrago \& Castañeda Lozano, 2011, p. 3). Del estudio de este derecho tal vez su justificación no se asocia a una motivación distributiva, sino tal vez, a un criterio de eficiencia. Lo anterior en cuanto que la paternidad de la innovación brinda información valiosa al mercado respecto de la reputación y capacidad intelectual de los agentes.

De acuerdo con Monroy (Análisis del modelo de las reglas de Calabresi y Melamed, 2011) "un derecho está protegido por una regla de inalienabilidad si no puedo transar y no puedo renunciar al derecho, como es el caso del derecho a la libertad". Al asociar esta explicación con los derechos morales se encuentra que se cumplen con las dos condiciones, a pesar de la libertad que se le da al agente innovador para que decida si desea o no ser nombrado, e incluso para que, como lo analiza Monroy, el agente pueda retractarse de su decisión (2011).

Ahora bien, se debe advertir que en este caso, resulta bastante cuestionable el por qué de la existencia de una regla de inalienabilidad en relación al derecho a la paternidad, puesto que como lo señala Monroy, acorde con las reglas de inalienabilidad de Calabresi y Melamed, no es clara la motivación que justifique la aplicación de esta regla. Si bien, puede existir una aparente motivación de eficiencia, también puede presentarse el caso en el cual el agente no desee ser mencionado y desee vender el derecho de paternidad, ante lo cual queda la duda de por qué no se puede hacer dicha transacción.

Hasta este punto se ha dado un vistazo a la catedral de la propiedad intelectual, de lo cual es posible evidenciar la existencia plena de reglas de propiedad, así como de responsabilidad en relación a la protección del conocimiento; así como también la existencia de una discutible regla de inalienabilidad. En la siguiente sección se dará paso a evaluar la eficiencia de tales reglas en relación a la función que cumple el conocimiento como factor productivo clave y fuente de bienestar para la sociedad.

\section{La catedral intelectual: una visión crítica a su diseño institucional}

Como se ha presentado hasta este punto, la propiedad intelectual se consolida como una herramienta fundamental para promover la generación de nuevo conocimiento. Esto a través de la incorporación de un sistema institucional que permite cambiar la característica natural de no exclusión del bien en análisis; lo cual respaldado en una serie de reglas de propiedad y responsabilidad, delinean la arquitectura para incentivar tal propósito.

Sin embargo, esta estructura institucional dista hoy en día de garantizar el equilibrio entre los intereses de los consumidores, que en condiciones de libre competencia esperan que los precios de los bienes sean aquellos donde los costos marginales se equiparen con los beneficios marginales, es decir, donde el mercado 
alcance su equilibrio. De manera intuitiva resulta evidente la contraposición de argumentos entre la búsqueda de un mercado que desde la perspectiva neoclásica se ajuste con el deber ser que supone el mercado perfecto, mientras en contrario se permite la existencia de una estructura que implica el desarrollo de estructuras de orden monopólico como lo son el sistema de patentes y los derechos de autor, bajo el argumento de propiciar la generación de un bien útil y necesario para la sociedad contemporánea.

En este orden, el argumento central de esta sección no se centra en discutir la eliminación o no del sistema de propiedad intelectual, sino en la necesidad de reajustar sus medios con la finalidad para la cual fue concebido inicialmente. Resulta indiscutible que la necesidad de recompensar el trabajo que implica la generación de más conocimiento, pero no así la forma como hoy en día se persigue tal finalidad.

Numerosos autores han reconocido abiertamente las limitaciones del sistema de propiedad intelectual, así como las externalidades negativas que de éste se derivan, Boyle (2003), Stiglitz J. E. (2008) ${ }^{8}$, Boldrin \& Levine (2008), Heller (2008), UNESCO (2005). Si se acepta que la versión contemporánea del sistema de propiedad intelectual tiene como fuente el estatuto de Monopolios de 1623 y la reina Anne de 1710 (Boldrin \& Levine, 2008, pp. 48 - 50), también sería interesante considerar como lo presentan Shavell \& Van Ypersele que la propagación en Europa de este sistema obedece más a un accidente político, que a un análisis concienzudo de los beneficios de su adopción (2001, p. 527).

Como lo plantea Correa (2011), este tipo de accidentes políticos son aún persistentes en la historia de la humanidad, la razón explicativa de la inclusión del tema de propiedad intelectual en el seno de la Organización es diseñada para promover el libre comercio, la Organización Mundial del Comercio (OMC). Correa (2011) señala cómo a juicio de un diplomático estadounidense los directores ejecutivos de Pfizer, Edmund T. Pratt, y John R. Opel de IBM, respectivamente, “(...) básicamente diseñaron, presionaron, y engañaron al gobierno para incluir la propiedad intelectual como uno de los puntos de la negociación" en el proceso de construcción de la $\mathrm{OMC}^{10}$.

8 Stiglitz manifiesta que cuando fue asesor del Consejo Económico, se opuso a que el acuerdo ADPIC, fuera parte de las negociaciones comerciales de la Ronda de Uruguay. Texto original en inglés: "When I was at the Council of Economic Advisors we opposed the Trade-Related Aspects of Intellectual Property Rights agreement (TRIPS), part of the Uruguay Round of trade negotiations" (Traducción libre del autor) (2008, p. 1694).

9 Texto original en inglés "“Pratt (Pfizer) and Opel (IBM) "basically engineered, pushed, and cajoled the government into including IP as one of the topics for the negotiation" (Traducción libre del autor).

10 De hecho, para algunas industrias como lo es el caso de la industria farmacéutica, el acuerdo ADPIC representa su mayor victoria, “(...) la cual desde que se lanzaron sus negociaciones en el marco de las negociaciones del Acuerdo General sobre Aranceles Aduaneros y Comercio (GATT por sus siglas en inglés) trabajó arduamente para expandir la protección de las patentes a aquellos de sus productos que en la mayoría de los países en desarrollo fueron excluidos de patentabilidad" (Correa, 2008, p. 11). 
En esta línea sería posible pensar en la existencia de aquello que la literatura de la nueva economía institucional ha denominado como la captura del regulador. Explicada como aquel fenómeno donde agentes organizados, también conocidos como grupos de presión, se comportan de manera estratégica para lograr que sus intereses prevalezcan sobre los de los demás grupos en contienda, con el objetivo último de lograr que sus reivindicaciones sean atendidas por el regulador (entidades estatales) y se materialicen en la generación de normas favorables para sus intereses (CETI, 1998).

Ahora bien, para poder entender las razones que llevan al rechazo de la inclusión de este tema dentro de las negociaciones tendientes a la liberalización del comercio, resulta necesario examinar los argumentos que se derivan de la estructura interna de este sistema.

Como se presentó al inicio de este análisis, la razón fundamental diseñar un sistema institucional por medio del cual se garantice la capacidad de exclusión sobre un bien público, fue la generación de incentivos para fomentar la producción de este bien, ello en respuesta a la gran cantidad de externalidades positivas que de éste se derivan. Sin embargo, la adopción de este criterio requiere lograr un equilibrio entre dos fenómenos, de los cuales hasta el momento solo uno, como comúnmente se hace, ha sido presentado.

Es así, como una visión completa de los retos que implica para el regulador la generación de un sistema que promueva la generación de conocimiento requiere el desarrollo de una estructura que permita balancear dos problemas, ello en ausencia de su captura. Estos dos problemas responden a dos tipos de ineficiencia connaturales a los bienes públicos, de un lado se encuentran las ineficiencias dinámicas, las cuales están asociadas con el suministro ineficiente, e implican la ausencia de incentivos para que las empresas innoven. En este escenario los consumidores pueden tener acceso a los bienes desarrollados a partir de nuevo conocimiento pagando un costo marginal tendiente a cero, o que podría considerarse casi simbólico pero, a su vez, se supone que la disponibilidad de bienes sería reducida puesto que serían pocos los agentes innovadores interesados en invertir sus recursos en esta actividad.

De otro lado se encuentran las ineficiencias estáticas, asociadas con problemas de subconsumo y las cuales, usualmente, quedan en un segundo plano de la discusión. Surgen como resultado de una decisión encaminada a solucionar la ineficiencia dinámica, para con ello aumentar la cantidad de bienes disponibles en el mercado (Stiglitz, 2000; 2008). En este caso, la ineficiencia se manifiesta de la siguiente manera: 
En este contexto el regulador deberá buscar el equilibrio entre los intereses del productor y los beneficios del consumidor, lo cual puede verse reducido a balancear, incentivos a la innovación vs. acceso a la innovación. Desafortunadamente, en la actualidad dicho equilibrio es inexistente. La UNESCO reconoce abiertamente el desequilibrio institucional derivado del sistema de propiedad intelectual. Así, en el año 2005, esta organización afirmó que:
"Según muchos expertos, es importante lograr un nuevo equilibrio entre la protección de los derechos de propiedad intelectual y la promoción del conocimiento de dominio público, habida cuenta del reforzamiento reciente de los dispositivos de protección - derecho de autor, y registro de invenciones $-\mathrm{y}$ del incremento de formas de piratería facilitadas por las nuevas tecnologías (...). (UNESCO, 2005, pp. 191 - 193).

El desequilibrio que advierte la UNESCO se manifiesta en profundas disparidades relacionadas, por ejemplo, con el acceso a medicamentos como una manifestación del derecho a la salud y los incentivos a la innovación para reconocer los recursos que se invierten para lograr un nuevo principio activo. En esta materia se encuentra como en un estudio adelantado por Hubbard \& Love (2004), se encontró que en periodo comprendido entre 1989 - 2000 se aprobaron por la Federal Drug Administration (FDA) 1035 nuevos medicamentos. Sin embargo de esos, solo el $23 \%$ presentó un avance terapéutico, menos del $1 \%$ se relacionaba con medicamentos para enfermedades huérfanas ${ }^{11}$. En lo que respecta al $76 \%$ restante solo presentó avances terapéuticos marginales o de poca consideración, lo cual deja en entredicho si los incentivos a la innovación están cumpliendo eficientemente su función.

En relación al reforzamiento de los dispositivos de protección, Heller(2008) advierte los efectos negativos de sistemas de propiedad intelectual más fuertes. En lo que él ha denominado como la tragedia de los anticomunes ${ }^{12}$ demuestra cómo el exceso de propiedad privada conduce a escenarios donde los costos de transacción se elevan a tal punto que resulta imposible llevar a cabo un acuerdo. Esta situación se encuentra muy bien ilustrada con lo que Goozner (2004) denominó como la

11 Respecto de los medicamentos huérfanos se puede decir que "A estos medicamentos se les llaman "huérfano" porque la industria farmacéutica tiene poco interés, bajo las condiciones normales del mercado, para desarrollar y poner en el mercado productos dirigidos solamente a una pequeña cantidad de pacientes que sufren de condiciones muy raras. Para las empresas medicamentosas, el coste de sacar al mercado un producto medicinal para una enfermedad rara no se recuperaría con las ventas esperadas del producto" (European Organization for rare diseases, 2009). La pequeña cantidad de pacientes corresponde a una tasa de menos de 5 pacientes por cada 10.000 habitantes.

12 Este nombre ha sido dado en contraposición a la tragedia de los comunes de Hardin(1968), la cual ha conducido a pensar equivocadamente que la única solución para los bienes públicos o los recursos comunes es la privatización, puesto que en ausencia de una regla de propiedad privada los agentes no tendrán incentivos para procurar el cuidado y renovación del bien público o del recursos común. 
tendencia en materia de propiedad intelectual en medicamentos en la década de los años noventa, "patentar primero y preguntar después" (2004, p. 350).

Heller ilustra la presencia de la tragedia señalada al analizar cómo se ha imposibilitado que un promisorio tratamiento para el Alzheimer sea probado, esto como resultado de la existencia de múltiples derechos de propiedad (patentes) que cada agente innovador cataloga como el más valioso o fundamental para el procedimiento y por lo cual exige su correspondiente pago. Lo anterior conduce a la configuración de una situación donde el costo de producir el tratamiento es superior a su beneficio económico (2008,pp. 4 - 6), conlleva esto a resultados obvios para el consumidor.

La situación descrita hasta este punto trae a primer plano la enorme responsabilidad que recae sobre el regulador, en cuanto a tratar de reequilibrar los derechos de consumidores y productores, los cuales se encuentran inmersos en el derecho a la libre competencia. Este derecho puede ser entendido como una articulación de medios y fines. En lo que respecta a los fines de este derecho, estos se encuentran consignados en el artículo 333 de la Constitución Política de 1991, donde se dice que "a libre competencia económica es un derecho de todos que supone responsabilidades"; en lo que respecta a los medios para lograr el goce de este derecho se encuentra la potestad que tiene el regulador para implementar un conjunto de herramientas bajo su tutela, para ocuparse "(...) de la protección de los intereses de los consumidores y de la protección de la libre competencia en los mercados" (SIC, 2008).

De lo anterior se deriva la importancia que tiene para el regulador la protección de la competencia en los mercados, esto a través de la generación de presión sobre las empresas, “(...) para que sean más eficientes y provean productos con mayor calidad y variedad a precios más bajos. Para esto, las empresas deben invertir en mejorar la calidad y producir más; incentivos directamente relacionados con el crecimiento económico y la innovación que se traducen en beneficios para los consumidores" (SIC, 2008).

Sin embargo, no resulta claro cómo se articulan las políticas de competencia, cuyo objetivo según la Organización Mundial de la Propiedad Intelectual (OMPI) es el de "asegurar el funcionamiento justo del mercado y, en particular, evitar que el acceso al mercado sea indebidamente obstaculizado o imposibilitado" (OMPI), con el sistema de propiedad intelectual en los términos expuestos en este documento. Para la OMPI, la articulación de estas dos instituciones gira en torno a dos ejes, “(...) por una parte, la legislación sobre patentes persigue luchar contra las copias o imitaciones de productos patentados, por lo que complementa las políticas de competencia para promover comportamientos equitativos en el mercado; por otra, las normas sobre competencia pueden limitar los derechos de patente al impedir que los titulares de patentes abusen de dichos derechos" (OMPI).

De la posición de la OMPI podría decirse que una legislación para fomentar la innovación puede adoptar dos vías, una orientada a garantizar su objetivo de promover la innovación y la generación de nuevo conocimiento útil para la 
sociedad, y otra centrada en garantizar las rentas monopolísticas que se generan por acción la estructura interna del sistema de propiedad intelectual. Así, claramente la lucha contra la piratería no contribuye directamente a la generación de nuevo conocimiento. De hecho, en una visión bastante discutible podría plantearse que la piratería se constituye, en ausencia de una estructura institucional balanceada, en uno de los pocos incentivos reales para la innovación en determinadas industrias, y que incluso en ciertas circunstancias brinda más beneficios que perjuicios, tal y como lo sostienen Balkin, Shepherd, \& De Castro (2004).

En lo que respecta a la posibilidad hacer uso de las normas de competencia para limitar el abuso de la posición de dominio que se puede originar al otorgar una patente, el caso del acceso a medicamentos para poblaciones con bajos recursos es el mejor ejemplo para ratificar el bajo grado de utilidad que tienen estas normas. Así, a pesar de que en el acuerdo ADPIC existían una serie de reglas de responsabilidad mencionadas en el anterior apartado, y las cuales fueron ratificadas a través de la Declaración Ministerial relativa al acuerdo sobre los ADPIC y la salud pública del 14 de Noviembre de 2001, la posibilidad de hacer uso de estas reglas de responsabilidad resulta casi inexistente ${ }^{13}$.

\section{Pilares para una nueva arquitectura intelectual}

A manera de conclusiones, este último apartado explora algunas alternativas propuestas para tratar de recuperar el equilibrio institucional entre consumidores y productores. Como punto de partida se debe reconocer que el sistema de propiedad intelectual ha pasado de ser un medio para incentivar la innovación a un fin en sí mismo, lo cual ha conducido a un evidente desequilibrio en su arquitectura.

En esta línea es necesario reconocer que los intereses socio económicos de "agentes innovadores" han primado sobre el derecho al acceso al conocimiento que tienen los consumidores. Frente a ello, no se trata de eliminar la regla de propiedad de los agentes innovadores y someter el derecho de propiedad a una regla de responsabilidad per se o inclusive prohibir cualquier tipo de transacción a través de una regla de inalienabilidad. Se trata de reordenar al interior la forma del diseño para tener un mejor aprovechamiento de éste, mediante el cual se beneficie y reconozca

13 Un caso interesante para estudiar en Colombia se relaciona con la venta del medicamento lopinavir ritonavir Kaletra ${ }^{\circledR}$ del laboratorio Abbott. En relación a este medicamento se solicitó en el año 2008 lo expedición de una licencia obligatoria, teniendo en cuenta entre otros argumentos, que mientras el precio de este medicamento para Colombia era de \$1683 USD por paciente al año, en compras institucionales en el país; en Ecuador el mismo medicamento se ofrecía a \$1000 USD por paciente al año; en Perú a un precio de \$744 USD por paciente año. Siendo el precio de venta en Colombia superior en dos o tres veces con respecto a sus vecinos (Mesa de organizaciones con trabajo en VIH SIDA; Fundación Henry Ardila; RECOLVIH; IFARMA; Acción esencial para la salud AIS; Essential Action; Misión Salud, 2008). 
el trabajo del agente innovador, así como el derecho legítimo de los consumidores a tener acceso al conocimiento. Más aún cuando es claro a que éste último se considera como la clave para cerrar la brecha entre aquellos que se consideran como desarrollados y los otros que están en proceso de desarrollo.

Retomadas las reglas de propiedad, responsabilidad e inalienabilidad expuestas en la sección II de este documento es posible evidenciar una serie de retos en la reconfiguración de la arquitectura intelectual. En relación al derecho de propiedad se considera necesario conservar los elementos temporal y cualitativo como criterios para determinar la asignación de este derecho.

El principal desafío para reestructurar la configuración de la arquitectura intelectual surge de la regla de propiedad expuesta en esa misma sección, la cual posibilita la exclusión en el uso de un bien público. Lo anterior atendidos los problemas derivados de este atributo, algunos de los cuales fueron mencionados en la sección III. En consideración a ello, resulta fundamental desvirtuar el mito que afirma que la única forma de lograr avances en ciencia, tecnología e innovación ha sido y es esta facultad de exclusión que brindan estructuras como las patentes o los derechos de autor. Al respecto, son numerosos los casos donde la innovación por competencia se muestra más eficiente ${ }^{14} \mathrm{y}$, a la vez, más consistente con el derecho a la libre competencia.

Tal vez uno de los ejemplos más emblemáticos es representado por el sistema operativo Linux, este como otros desarrollos en materia de software libre ha demostrado que la construcción de conocimiento a través de una lógica cooperativa en donde cada individuo busca maximizar su utilidad personal es posible sin que ello implique una desmejora en la situación de los demás. En otros términos, podría asumirse que la lógica de trabajo que subyace al software libre logra un óptimo de Pareto.

De hecho, como lo plantea Eric S. Raymon, en su artículo La catedral y el bazar:

"El mundo de Linux se comporta en muchos aspectos como el libre mercado o un sistema ecológico, donde un grupo de agentes individualistas buscan maximizar la utilidad en la que los procesos generan un orden espontáneo autocorrectivo más desarrollado y eficiente que lo que podría lograr cualquier tipo de planeación centralizada" (Raymond, 1998).

La lógica del modelo que entraña el software libre, demuestra cómo sin la existencia de exclusión en el conocimiento es posible avanzar en innovación y desarrollo. No obstante, esto no implica que los agentes innovadores no sean recompensados por su esfuerzo. Para el caso de Linux la función de utilidad “(...)

14 Una fuente interesante de casos se encuentra en el texto de Boldrin \& Levine (2008) "Against Intellectual Monopoly", editado por Cambridge Press y disponible para su consulta en la siguiente dirección electrónica: http://www.dklevine. com/general/intellectual/againstfinal.htm 
no es económica en el sentido clásico, sino algo intangible como la satisfacción de su ego y su reputación entre otros hackers. (Uno podría hablar de su "motivación altruista", pero ignoraríamos el hecho de que el altruismo en sí mismo es una forma de satisfacción del ego para el altruista). Los grupos voluntarios que funcionan de esta manera no son escasos realmente; uno en el que he participado es el de los aficionados a la ciencia ficción, que a diferencia del mundo de los hackers, reconoce explícitamente el "egoboom" (el realce de la reputación de uno entre los demás) como la motivación básica que está detrás de la actividad de los voluntarios" (Raymond, 1998).

Ahora bien, desde el punto estrictamente económico se reconoce la existencia de una serie de beneficios e incentivos para la innovación bajo un sistema de competencia, es así como las

“(...) ventajas que otorga "moverse primero" (conseguir externalidades de red, liderar la curva de aprendizaje, estimular la lealtad a la marca, imponer coste de cambio al consumidor y crear el estándar del producto, entre otros) resultan mucho más determinantes que los beneficios empresariales que la prohibición legal de imitar que la patente introduce" (Ortún Rubio, 2004, pp. 194 - 195).

Así, la nueva regla de propiedad debe además de conciliar los beneficios de acceder a más conocimiento, a mejores y más bienes para el consumidor, es decir, la mejoría de su bienestar, con la retribución por el trabajo del agente innovador, debe generar de incentivos para la cooperación.

El cambio en la regla de propiedad tiene efectos directos sobre la estructuración de la regla de responsabilidad. Al respecto en el caso relacionado con la piratería esta conducta tendería a desaparecer pero, no por ello los efectos negativos para el consumidor que se pueden generar con la venta de bienes y servicios que no cumplen con los estándares de calidad, o que en el caso de las falsificaciones de medicamentos pueden comprometer la vida de un individuo. Frente a ello la regla de responsabilidad debería estructurarse para compensar el daño que pueda sufrir el consumidor y así de manera indirecta beneficiar al productor, y no como hoy en día se aplica que favorece al productor y de manera indirecta al consumidor. La estructuración de esta regla de responsabilidad en los términos antes mencionados lleva la discusión a una arena diferente, el derecho del consumidor.

En lo que respecta a la segunda regla de responsabilidad, relacionada con la posibilidad de expedir licencias obligatorias, que como se mencionó esta facultad no tiene aplicación práctica hoy en día, se configuraría una situación que lleva a que la regla de responsabilidad no tenga justificación, puesto que la licencia obligatoria en los términos de los artículos 61, 65 y 66 de la Decisión 486, sólo se aplica sobre el conocimiento protegido vía patentes, es decir, sobre el conocimiento que puede ser excluible de su uso.

Finalmente, en lo relacionado con la regla de inalienabilidad asociada con el derecho moral del agente innovador, queda el debate abierto sobre la eficiencia o no 
de permitir la transacción de este derecho, sin embargo, a la luz de los incentivos que deberían contemplarse bajo la nueva regla de propiedad sería pertinente no perder de vista la utilidad que genera para el mercado conocer la fuente de la innovación.

\section{REFERENCIAS}

Balkin, D. B., Shepherd, D. A., \& De Castro, J. (2004). Piracy as strategy?: A reexamination of product piracy. IE Working Paper , 1 - 19.

Boldrin, M., \& Levine, D. K. (2008). Against Intellectual Monopoly. New York: Cambridge University Press.

Boyle, J. (2003). The second enclosure movement and the construction of the public domain. Law and contemporary problems, 66 (1-2), 33-74.

Buitrago, J. D., \& Castañeda Lozano, M. I. (2011). La función social como límite a la propiedad intelectual. Bogotá D.C.: Mimeo.

Calabresi, G., \& Melamed, A. D. (1972). Reglas de propiedad, reglas de responsabilidad y de inaliebabilidad: una vista de la cátedral. Harvard Law Review, 85 (6), 1089 $-1128$.

Castro García, J. D. (2009). la propiedad industrial. Bogotá: Universidad Externado de Colombia.

CETI. (1998). Marco teoríco de la regulación. Revista Regulación, 4 - 47.

Comunidad Andina. (2000). Decisión 486: Régimen Común de Propiedad Industrial. Lima: La Comisión de la Comunidad Andina.

Cooter, R., \& Ulen, T. (1997). Derecho y economía (Primera reimpresión ed.). México, México: Fondo de Cultura Económica.

Correa, C. M. (2008). Intellectual Property Rights and Inequalities in Health Outcomes. Globalization and Health Knowledge Network, 28.

Correa, C. M. (Octubre de 2011). Los factores económicos en el diseño de políticas de propiedad intelectual en países en desarrollo. Bogotá D.C., Bogotá D.C., Colombia.

European Organization for rare diseases. (19 de Agosto de 2009). ¿Qué es un medicamento huérfano? Recuperado el 8 de Noviembre de 2011, de EURODIS: Rare Diseases Europe: http://www.eurordis.org/es/content/ique-es-unmedicamento-huerfano

Furobont, E., \& Pejovich, S. (1972). Property rights and economic theory: a survey of recent literature. Journal of economic literature, 10 (5), 1137-1162.

Goozner, M. (2004). La píldora de los 800 millones de dólares: la verdad sobre el costo de las nuevas drogas.Bogotá: Grupo Editorial Norma.

Hardin, G. (1968). The tragedy of the Commons. Science, 162, 1243-1248.

Heller, M. (2008). The Gridlock economy: How too much ownership wrecks markets, stops innovation, and costs lives. New York: Basic Books. 
Hubbar, T., \& Love, J. (2004). A new trade framework for global healthcare R\&D. PlosBiology, 2 (2), 147 - 150.

Locke, J. (2009). Segundo Tratado sobre el Gobierno Civil: Un ensayo acerca del verdadero origen, alcance y fin del Gobierno Civil (Quinta reimpresión ed.). (C. Mellizo, Trad.) Madrid: Alianza Editorial.

Mankiw, N. G. (1998). Principios de Economía (Primera ed.). Madrid: McGraw Hill. Mesa de organizaciones con trabajo en VIH SIDA; Fundación Henry Ardila; RECOLVIH; IFARMA; Acción esencial para la salud AIS; Essential Action; Misión Salud. (2008). Documento técnio: Solicitud de una licencia obligatoria abierta para Lopinavir/Ritonavir (Kaletra) por motiviso de interés público en la República de Colombia. Bogotá D.C.: Mimeo.

Monroy Cely, D. A. (2 de 11 de 2011). Análisis del modelo de las reglas de Calabresi y Melamed. (M. A. Pinzón Camargo, Entrevistador)

Monroy Cely, D. A. (2010). Análisis económico de la intervención judicial en los contratos: ¿Una cuestión de justicia? Working paper, 44.

OMC. (1994). Anexo 1C Acuerdo sobre los aspectos de los derechos de propiedad intelectual relacionados con el comercio. Acuerdo por el que se establece la $O M C$.

OMC. (2011). Entender la OMC: lo que propugnamos. Recuperado el 7 de 11 de 2011, de Organización Mundial del Comercio: http://www.wto.org/spanish/ thewto_s/ whatis_s/what_stand_for_s.htm

OMPI. (s.f.). La competencia y las patentes. Recuperado el 1 de 11 de 2011, de Organización Mundial de la Propiedad Intelectual: http://www.wipo.int/ patentlaw/es/developments/competition.html

Ortún Rubio, V. (2004). Patentes, regulación de precios e innovación en la industria farmacéutica. Cuadernos Económicos de ICE (67), 191-207.

Pinzón Camargo, M. A. (2010). La Carrera entre los microbios, los virus y la investigación: Un conflicto de instituciones. En Colección Enrique Low Murtra: Derecho y Economía (Vol. VII, pp. 185-214). Bogotá D.C.: Universidad Externado de Colombia.

Raymond, E. S. (28 de Julio de 1998). La Catedral y el Bazar. Recuperado el 8 de Noviembre de 2011, de Biblio Web de Sin Dominio: telematica/catedral.html

Samuelson, P. A., \& Nordhaus, W. D. (1999). Economía. Barcelona: McGraw Hill.

Shavell, S., \& Van Ypersele, T. (2001). Rewards versus intellectual property rights. Journal of Law and Economics, XLIV, 525-547.

SIC. (2008). Definiciones sobre competencia. Recuperado el 8 de 11 de 2011, de Superintendencia de Industria y Comercio: http://www.sic.gov.co/en/web/guest/ libre-competencia;jsessionid=905D651ED0FA6FCBB1578999 1854CBFF

SIC. (2008). Patente de modelos de utilidad: ¿Existe otra clase de patente? Recuperado el 31 de 10 de 2011, de Superintendencia de Industria y Comercio: http://www.sic.gov.co/en/web/guest/modelos-de-utilidad1 
SIC. (2008). Patentes: ¿Cómo solicitar una patente? Recuperado el 31 de 10 de 2011, de Superintendencia de Industria y Comercio: http://www.sic.gov.co/en/web/ guest/patentes

Stiglitz, J. E. (2008). Economic foundations of intellectual property. Duke Law Journal, 57 (6), 1693-1724.

Stiglitz, J. E. (2000). La economía del sector público (Tercera ed.). (M. E. Rabasco, \& L. Toharia, Trads.) Barcelona: Antoni Bosch.

Stiglitz, J. (1999). Knowledge as a Global Public Good. En I. Kaul, I. Grunberg, \& M. A. Stern (Edits.), Global Public Goods: International Cooperation in the 21st Century (págs. 308 - 325). Oxford University Press.

UNESCO. (2005). Hacia las sociedades del conocimiento. París: UNESCO. 